\title{
Chromomagnetism, flavour symmetry breaking and S-wave tetraquarks
}

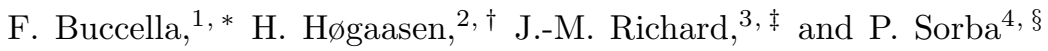 \\ ${ }^{1}$ Università di Napoli Federico II, Dipartimento di Scienze Fisiche and INFN, Sezione di Napoli, \\ Complesso Universitario di Monte Sant' Angelo, via Cintia I-80126 Napoli, Italy \\ ${ }^{2}$ Department of Physics, University of Oslo, Box 1048 NO-0316 Oslo, Norway \\ ${ }^{3}$ Laboratoire de Physique Subatomique et Cosmologie, Université Joseph Fourier-IN2P3-CNRS \\ 53, avenue des Martyrs, 38026 Grenoble cedex, France \\ ${ }^{4}$ Laboratoire d'Annecy-le-Vieux de Physique Théorique (LAPTH) \\ 9, chemin de Bellevue, B.P. 110, 74941 Annecy-le-Vieux Cedex, France
}

(Dated: October 30, 2018)

\begin{abstract}
The chromomagnetic interaction, with full account for flavour-symmetry breaking, is applied to S-wave configurations containing two quarks and two antiquarks. Phenomenological implications are discussed for light, charmed, charmed and strange, hidden-charm and double-charm mesons, and extended to their analogues with beauty.
\end{abstract}

PACS numbers: 12.39.-x,12.39.Mk,12.40.Yx

\section{INTRODUCTION}

The question of the existence of multiquark hadrons beyond ordinary mesons and baryons has been addressed since the beginning of the quark model. It has been particularly discussed recently with the firm or tentative discovery of new hadron states in a variety of experiments. For a review of recent results, see, e.g., Refs. 1, 2, [3, 4].

Different mechanisms have been proposed to form stable or metastable multiquarks in the ground state. The most natural mechanism, especially for states close to a hadron-hadron threshold, is provided by nuclear forces, extrapolated from the nucleon-nucleon interaction, and acting between any pair of hadrons containing light quarks. This led several authors to predict the existence of $\mathrm{DD}^{*}$ and $\mathrm{D}^{*} \overline{\mathrm{D}}(+$ c.c.) molecules $[\underline{5}, 6,6,7,8,8,9]$. According to these authors (see, also, [10, 11, 12]), the latter configuration is perhaps seen in the $\mathrm{X}(3872)$ 13], though other interpretations have been proposed for this narrow meson resonance with hidden charm [14, 15]. Stable or metastable multicharmed dibaryons are also predicted in this nuclear-physics type approach [16].

Flavour independence is a key property of QCD, at least in the heavy-quark limit. Quarks are coupled to the gluon field through their colour, not their mass, and this induces a static interquark potential which is independent of the flavour content, in the same way as the same Coulomb interaction is kept acting on antiprotons, kaons, muons and electrons when exotic atoms and molecules are studied [17]. The mechanism by which the hydrogen molecule is more deeply bound than the positronium molecule remains valid, mutatis mutandis, in hadron physics with flavour independence and favours the binding of $(Q Q \bar{q} \bar{q})$ below the threshold of two heavy-flavoured mesons, when the quark-mass ratio $Q / q$ increases [18, 19, 20, 21, 22, 23, 24, 25].

The best known mechanism for multiquark binding is based on spin-dependent forces. In the late 70's, Jaffe [26, 27] proposed a $\left(q^{2} \bar{q}^{2}\right)$ picture of some scalar mesons, as a solution to the puzzle of their low mass, decay and production properties, and abundance. He also discovered that the colour-spin operator entering the widelyaccepted models sometimes provides multiquark states with a coherent attraction which is larger than the sum of the attractive terms in the decay products, hence favouring the formation of bound states. An example is the so-called $\mathrm{H}$ dibaryon [28], with spin $S=0$ and quark content (ssuudd), tentatively below any threshold

\footnotetext{
*Electronic address: buccella@na.infn.it

${ }^{\dagger}$ Electronic address: hallstein.hogasen@fys.uio.no

‡Electronic address: jean-marc.richard@lpsc.in2p3.fr

$\S$ Electronic address: sorba@lapp.in2p3.fr
} 
made of two light baryons. This prediction stimulated an intense experimental work, which did not lead to any positive evidence, see, e.g., [29].

This model for the $\mathrm{H}$ also provoked much theoretical activity. New configurations were found, in which the chromomagnetic effects are favourable, such as the 1987-vintage pentaquark [30, 31], $\mathrm{P}=\left(Q \bar{q}^{4}\right)$. A comprehensive systematics of multiquark configurations with favourable chromomagnetic effects can be found, e.g., in [32, 33, 34] and the references therein.

In the early days of multiquark investigations, chromomagnetic effects were also intensively used in an attempt to explain the narrow hadronic resonances which were observed at that time [35]. Models were proposed for these hadrons, with two clusters of complementary colour separated by an orbital momentum, to prevent the decay into colour singlets without pair creation and thereby give possible long lived states [36, 37, 38, 39]. The effective mass of each cluster was computed from the chromomagnetic interaction and effective quark masses. The most studied states were the tetraquarks called "baryonium" and the pentaquarks called "mesobaryonium".

In the limit of exact $\mathrm{SU}(\mathrm{N})_{\mathrm{F}}$ flavour-symmetry, the chromomagnetic model reads

$$
H=\sum_{i} m_{i}-C \sum_{i<j} \tilde{\lambda}_{i} \cdot \tilde{\lambda}_{j} \boldsymbol{\sigma}_{i} \cdot \boldsymbol{\sigma}_{j},
$$

where $\boldsymbol{\sigma}_{i}$ is the spin operator and $\tilde{\lambda}_{i}$ the colour operator acting on the $i^{\text {th }}$ quark, and each effective mass $m_{i}$ includes the constituent quark mass and its chromoelectric energy (binding effect). There is already an abundant literature on how to estimate the expectation value of the chromomagnetic operator for multiquark configurations, in particular using some powerful group-theoretical techniques. The Hamiltonian (1) is expressed in terms of Casimir operators of the spin, colour and spin-colour groups. When the overall strength factor $C$ is replaced by a coupling $C_{i j}$ which depends on the quark flavour, an explicit basis is required to estimate the eigenstates of $H$.

Note that the role of $\mathrm{SU}(3)_{\mathrm{F}}$ symmetry breaking has already been analysed in the literature, see, in particular, [40, 41, 42, 43] for the $\mathrm{H}$ and the $\mathrm{P}$. It often happens that the corrections weaken or even spoil the binding predicted in the $\mathrm{SU}(3)_{\mathrm{F}}$ limit.

In this paper, a detailed formalism is presented to fully account for flavour-symmetry breaking in the chromomagnetic interaction, and an application is given to the sector of systems made of two quarks and two antiquarks in a relative $\mathrm{S}$-wave, i.e., scalar $\left(J^{P}=0^{+}\right)$, axial $\left(1^{+}\right)$and tensor $\left(2^{+}\right)$mesons. The question then is how to extrapolate the strength of the chromomagnetic interaction from the meson or baryon sector to the case of multiquark configurations.

There has been several investigations of multiquark states using the remarkable know-how of few-body physics. The strategy here consists in writing down an explicit Hamiltonian with kinetic energy operator, spin-independent confining forces and spin-dependent terms, tuning the parameters to reproduce some known mesons and baryons, and solve the multiquark problem. This involves an extrapolation of the linear quarkantiquark potential toward multiquark states and an ad-hoc regularisation of the contact interaction, which then can be treated beyond first order.

The present approach is somewhat complementary. The role of the chromomagnetic interaction is analysed from the point of view of the symmetry properties, to deduce patterns shared by a whole class of models. The study is restricted to the chromomagnetic model, though it has been challenged recently by models where the hyperfine splittings of hadrons is described by instanton-induced forces or spin-flavour terms. The multiquark sector in these models is reviewed by Stancu [44] or Sakai et al. [43].

It is well known that a colour singlet configuration with two quarks and two antiquarks has at least one component which is a product of two colour singlets. Hence most states are very broad, since unstable against spontaneous dissociation, and give only indirect signatures. However, in rare circumstances, the dissociation is kinematically suppressed, resulting into a remarkably small width. This is the scenario proposed recently for the $\mathrm{X}(3872)$ [15].

The applications will be focused on four-quark mesons with spin $S=0,1$ or 2 , and various flavour content. As already mentioned, there are promising possibilities in the exotic sector with two heavy quarks, especially $(b c \bar{q} \bar{q})$, but these states have not yet been experimentally searched for. However, there are indications of supernumerary states in the charmonium spectrum [4, 13, 45, 46]. The single-charm states $(c q \bar{q} \bar{q})$ and analogues with strangeness were predicted many years ago [47], and the recent findings in the $\mathrm{D}_{s}$ spectrum might reveal some of these sates.

The hottest sector is the one of scalar mesons. Recent experiments at LEAR and at B-factories have confirmed the years of data taking and analysis: there are far too many scalar mesons below $2 \mathrm{GeV}$ for the only $q \bar{q}(q$ 
denotes $u$ or $d$ ) and $s \bar{s}$ states, even including the radial excitations. The fashion evolved from the multiquarks of Jaffe to glueballs and hybrids, but seemingly tends again toward multiquarks. It is hardly possible to propose an ultimate solution to this problem. It appears clearly from the detailed phenomenological analyses [48, 49, 50, 51, 52] that states with different quark and gluon content are abundantly mixed and acquire an appreciable mass shift due to their coupling to the real or virtual decay channels. Nevertheless, such a mixing should operate between properly identified bare states, and some clarification will be suggested in the four-quark sector which is a key ingredient of the mixing scheme.

This paper is organised as follows: in Sec. III the most general chromomagnetic Hamiltonian is presented and diagonalised for systems of two quarks and two antiquarks. The application to various flavour sectors is presented in Sec. III before the conclusions in Sec. IV]

\section{THE CHROMOMAGNETIC HAMILTONIAN}

\section{A. General considerations}

The interaction Hamiltonian acting on the colour and spin degrees of freedom, and generalising (1), is

$$
H=\sum_{i} m_{i}+H_{\mathrm{CM}}, \quad H_{\mathrm{CM}}=-\sum_{i, j} C_{i j} \tilde{\lambda}_{i} \cdot \tilde{\lambda}_{j} \boldsymbol{\sigma}_{i} \cdot \boldsymbol{\sigma}_{j} .
$$

It is inspired by one-gluon-exchange [53], in which case $C_{i j}$ contains a factor $\alpha_{s} /\left(m_{i} m_{j}\right)$, where $\alpha_{s}$ is the coupling constant of QCD and $m_{i}$ the mass of the $i^{\text {th }}$ quark, and also the probability of finding the quarks (or antiquarks) $i$ and $j$ at the same location. The above model is more general. The coefficients $C_{i j}$ which presumably incorporate non-perturbative QCD contributions, depend on the quark masses and on the properties of the spatial wave function, as in the one-gluon-exchange model. The solution of the eigenvalue problem for the Hamiltonian (2) is of interest not only for spectroscopy, but in all circumstances where two quarks and two antiquarks are in a relative S-wave, for instance when studying the violation of the OZI rule [54].

For a quark-antiquark meson, $\left\langle\tilde{\lambda}_{1} \cdot \tilde{\lambda}_{2}\right\rangle=-16 / 3$ and $\sigma_{1} \cdot \sigma_{2}=+1$ for spin $S=1$ and -3 for $S=0$. The Hamiltonian (2) accounts naturally for the observed hyperfine splittings such as $\mathrm{J} / \psi-\eta_{c}$ or $\mathrm{D}_{s}^{*}-\mathrm{D}_{s}$. This leads to the strength parameters shown in Table [. As the spin-singlet state of bottomonium and the spin-triplet state of $(b \bar{c})$ are not yet known experimentally, and in these sectors, the data have been replaced by model calculations [55].

TABLE I: Values of $C_{q^{\prime} \bar{q}}$ (in $\mathrm{MeV}$ ) estimated from meson masses

\begin{tabular}{lrlll}
\hline \hline & $n$ & $s$ & $c$ & $b$ \\
\hline $\bar{n}$ & 29.8 & & & \\
$\bar{s}$ & 18.4 & 8.6 & 5.5 & $4.1^{a}$ \\
$\bar{c}$ & 6.6 & 6.7 & $6.8^{a}$ & 2.2 \\
$\bar{b}$ & 2.1 & 2.2 & \\
\hline \hline
\end{tabular}

${ }^{a}$ This is extracted from one of the model calculations compiled in Ref. [55]

For ordinary baryons, the colour operator $\tilde{\lambda}_{i} \cdot \tilde{\lambda}_{j}=-8 / 3$ is the same for all pairs and factors out. For spin $S=3 / 2, H_{\mathrm{CM}}=8\left(C_{12}+C_{23}+C_{31}\right) / 3$ pushes up $\Delta, \Sigma^{*}$, etc. For spin $S=1 / 2\left(q q q^{\prime}\right)$ baryons with two identical quarks, $H_{\mathrm{CM}}=8 / 3\left(C_{12}-4 C_{13}\right)$ is attractive. In the general case $\left(q_{1} q_{2} q_{3}\right)$ of spin $1 / 2$ such as $\Lambda$ or $\Sigma_{0}$ with breaking of isospin symmetry, or $\Xi_{c}^{+}(c s u)$, a basis

$$
\left[\left(q_{1} q_{2}\right)_{1} q_{3}\right]_{1 / 2}, \quad\left[\left(q_{1} q_{2}\right)_{0} q_{3}\right]_{1 / 2},
$$

can be chosen, with symmetric or antisymmetric coupling of the first two quarks (the index, here and in similar further states, denotes the value of the spin) in which the chromomagnetic interaction reads

$$
H_{\mathrm{CM}}=\frac{8}{3}\left[\begin{array}{cc}
C_{12}-2 C_{13}-2 C_{23} & \sqrt{3}\left(C_{23}-C_{13}\right) \\
\sqrt{3}\left(C_{23}-C_{13}\right) & -3 C_{12}
\end{array}\right] .
$$


The $\mathrm{N}-\Delta$ system gives access to $C_{q q}$. Then the $\Lambda-\Sigma-\Sigma^{*}$ multiplet gives $C_{q s}$ and another value of $C_{q q}$ close to the previous one. Then $\left\{\Xi, \Xi^{*}\right\}$ and $\Omega^{-}$depend on $m_{s}+4 C_{s s} / 3$ and $m_{s}+8 C_{s s} / 3$ and, to the extent that these parameters do not change much from $\Xi$ to $\Omega, C_{s s}$ can be obtained. The value shown for $C_{c c}$ is from model calculations of double-charm baryons [56]. The values of the strength factors $C_{i j}$ are displayed in Table III.

TABLE II: Approximate values of $C_{i i^{\prime}}$ (in $\mathrm{MeV}$ ) estimated from baryon masses

\begin{tabular}{lrrr}
\hline \hline & $n$ & $s$ & $c$ \\
\hline$n$ & {$[19-20]$} & {$[5-10]$} & \\
$s$ & {$[12-14]$} & 5 & $5^{b}$ \\
$c$ & 4 & 5 & \\
\hline \hline
\end{tabular}

${ }^{b}$ This is extracted from one of the model calculations in Ref. [56]

For tetraquarks and higher multiquark states, there is the known complication that an overall colour singlet can be built from several manners of arranging internal colour. These colour states usually can mix and one has to diagonalise the interaction Hamiltonian. In the case of tetraquarks, the most natural basis is constructed by coupling the quarks $q_{1}$ and $q_{2}$ in colour $\overline{3}$ or 6 and spin $s=0$ or 1 , to the extent allowed by the Pauli principle, and similarly for the antiquarks. However, for studying the decay properties, it is convenient to translate the state content in the basis $\left[\left(q_{1} \bar{q}_{3}\right)^{c}\left(q_{2} \bar{q}\right)^{c}\right]$ or $\left[\left(q_{1} \bar{q}_{4}\right)^{c}\left(q_{2} \bar{q}_{3}\right)^{c}\right]$. Here, and in the rest of this article, the upper index $c$ denotes the colour of the cluster. It runs over $c=1$ and $c=8$ in this decomposition. The relevant crossing matrices should be derived with care, as some errors and misprints occurred in the early literature. In particular, the order adopted for coupling $q_{1}$ and $q_{2}$, for instance, results into phase factors that do not influence the physics content, but should be treated consistently throughout the calculation. The results presented below have been checked in particular against [57] in the limit of isospin symmetry, and [58].

\section{B. Group theoretical considerations}

The operator $\mathcal{O}=-\sum \tilde{\lambda}_{i} \cdot \tilde{\lambda}_{j} \boldsymbol{\sigma}_{i} \cdot \boldsymbol{\sigma}_{j}$ can be elegantly expressed in terms of the Casimir operators of the spin $\mathrm{SU}(2)_{\mathrm{s}}$, colour $\mathrm{SU}(3)_{\mathrm{c}}$ and spin-colour $\mathrm{SU}(6)_{\mathrm{cs}}$ groups, as stressed in [27, 39, 59] for special configurations or more general cases.

For an $N$-constituent system consisting of $n$ quarks and $\bar{n}=N-n$ antiquarks, with the same strength $C_{i j}=C$ in the quark sector, $C_{i j}=\bar{C}$ in the antiquark sector, and $C_{i j}=C^{\prime}$ for all quark-antiquark pairs, it can be shown that

$$
\begin{aligned}
& 2 H_{\mathrm{CM}}=-C\left[C_{6}(Q)-C_{3}(Q)-\frac{8}{3} C_{2}(Q)-16 n\right]-\bar{C}\left[C_{6}(\bar{Q})-C_{3}(\bar{Q})-\frac{8}{3} C_{2}(\bar{Q})-16 \bar{n}\right] \\
& +C^{\prime}\left[C_{6}(T)-C_{6}(Q)-C_{6}(\bar{Q})-C_{3}(T)+C_{3}(Q)+C_{3}\left(\bar{Q}-\frac{8}{3} C_{2}(T)+\frac{8}{3} C_{2}(Q)+\frac{8}{3} C_{2}(\bar{Q})\right],\right.
\end{aligned}
$$

where $C_{2}, C_{3}$ and $C_{6}$ are the Casimir operators of $\mathrm{SU}(2)_{\mathrm{s}}, \mathrm{SU}(3)_{\mathrm{c}}$ and $\mathrm{SU}(6)_{\mathrm{cs}}$, respectively, for the quark $(Q)$ or antiquark $(\bar{Q})$ sector or the whole system $(T)$. The normalisation adopted here is such that $C_{2}=S(S+1)$ for a spin $S$, and $C_{3}(3)=16 / 3$ and $C_{6}(6)=70 / 3$ for the lowest representations. If it is further assumed that $C=\bar{C}=C^{\prime}$, the well-known formula [27]

$$
\mathcal{O}=8 N+\frac{1}{2} C_{6}(T)-\frac{4}{3} C_{2}(T)-\frac{1}{2} C_{3}(T)+C_{3}(Q)+\frac{8}{3} C_{2}(Q)-C_{6}(Q)+C_{3}(\bar{Q})+\frac{8}{3} C_{2}(\bar{Q})-C_{6}(\bar{Q})
$$

is recovered.

It is possible to make some general considerations on the eigenvalues of the chromomagnetic interaction for the scalar, axial and tensor tetraquarks. Consider first the flavour-symmetry limit, which is a good approximation for the states built from light $(q=u, d)$ quarks and antiquarks. In this limit, the matrix representation $H_{\mathrm{CM}}$ simplifies to two $2 \times 2$ matrices for the scalars, two $2 \times 2$ and two $1 \times 1$ for the axials and two $1 \times 1$ for the 
tensors. The interaction between the quarks and the antiquarks, which depends strongly on the SU(6) Casimir operators of the tetraquark, has a tendency to give eigenstates which approximately belong to the irreducible representations of that algebra.

This observation has also interesting consequences for the decay properties of tetraquarks. In fact, many years ago, Jaffe [26, 27] stressed that all the multiquarks have "open door" channels, that is to say, can decay into two colour singlets by simple rearrangement of the constituents, see, also, Refs. 60, 61]. Only phase space can possibly block this spontaneous dissociation.

More recently, this property has been related 62] to the transformation properties of the multiquark states with respect to $\mathrm{SU}(6)_{\mathrm{cs}}$. Since the pseudoscalar $\left(\pi, \mathrm{K}, \eta, \eta^{\prime}\right)$ and the vector $\left(\rho, \mathrm{K}^{*}, \omega, \phi\right)$ mesons transform as a singlet and a 35, respectively, the "open door" pseudoscalar-pseudoscalar (PP) channels will be SU(6) singlets and the pseudoscalar-vector (PV) channels will be 35-plets of the same algebra. The "open door" vector-vector (VV) channels will be found for the states transforming in a representation contained in the product of two 35 representations $(1,35,189,280, \overline{280}$ and 405$)$.

The scalar states built from light quarks belong to the representations $1+405$ of $\mathrm{SU}(6)_{\mathrm{cs}}$ for the case of isospin $I=0$ and to the representations $1+189$ for $I=0,1,2$. Indeed, the quarks symmetric (resp. antisymmetric) in colour-spin belong to the $(6 \times 6)_{\mathrm{S}}=21$ (resp. $(6 \times 6)_{\mathrm{A}}=15$ representations of $\mathrm{SU}(6)_{\mathrm{cs}}$. From the decomposition of the $\mathrm{SU}(6)_{\mathrm{cs}}$ representations with respect to $\mathrm{SU}(3)_{\mathrm{c}} \times \mathrm{SU}(2)_{\mathrm{s}}$

$$
21=(6,3)+(\overline{3}, 1), \quad 15=(6,1)+(\overline{3}, 3)
$$

and the $\mathrm{SU}(6)_{\mathrm{cs}}$ products of representations

$$
21 \times \overline{21}=1+35+405, \quad 15 \times \overline{15}=1+35+189 .
$$

it is readily seen that two $(1,1)$ singlets of $\mathrm{SU}(3)_{\mathrm{c}} \times \mathrm{SU}(2)_{\mathrm{s}}$ come from the $21 \times \overline{21}$ and $15 \times \overline{15}$ products, and also that the 35 representation does not contain any $(1,1)$ singlet of $\mathrm{SU}(3)_{\mathrm{c}} \times \mathrm{SU}(2)_{\mathrm{s}}$.

In order to apply Eqs. (6) and (5) to these states, the following SU(6) Clebsch-Gordan coefficients are necessary

$$
\begin{aligned}
|1\rangle & =\sqrt{\frac{6}{7}}|21 ;(6,3)\rangle|\overline{21} ;(\overline{6}, 3)\rangle+\frac{1}{\sqrt{7}}|21 ;(\overline{3}, 1)\rangle|\overline{21} ;(3,1)\rangle, \\
|405\rangle & =\frac{1}{\sqrt{7}}|21 ;(6,3)\rangle|\overline{21} ;(\overline{6}, 3)\rangle-\sqrt{\frac{6}{7}}|21 ;(\overline{3}, 1)\rangle|\overline{21} ;(3,1)\rangle, \\
|1\rangle & =\sqrt{\frac{2}{5}}|15 ;(6,1)\rangle|\overline{15} ;(\overline{6}, 1)\rangle+\sqrt{\frac{3}{5}}|15 ;(\overline{3}, 3)\rangle|\overline{15} ;(3,1)\rangle, \\
|189\rangle & =\sqrt{\frac{3}{5}}|15 ;(6,1)\rangle|\overline{15} ;(\overline{6}, 1)\rangle-\sqrt{\frac{2}{5}}|15 ;(\overline{3}, 3)\rangle|\overline{15} ;(3,1)\rangle .
\end{aligned}
$$

Now, the SU(6) $)_{\text {cs }}$ Casimir dependence of the chromomagnetic contribution to the mass of the tetraquarks shown in Eq. (9) implies that the lightest states are approximately singlets, while the heavier states transforming approximately as the 405 or the 189 representation, have large coupling to VV and small coupling to PP channels.

As for the axial sector, the lightest state will be a $I=0$ transforming as a 35 , followed by two $I=1$ states and a $I=0,1,2$ cluster transforming in the same way, while the heaviest states are the two $I=1$ transforming approximately as $280+\overline{280}$. Due to parity conservation, a $1^{+}$state cannot decay into two pseudoscalar mesons, the heaviest states are expected to have a small amplitude to PV and may lie below the threshold for VV. Note that the four 35 may be too light to decay into PV.

Finally, the tensor states, which have S-wave amplitudes into VV, may be under threshold for that final state.

When states with one or more strange constituents are considered, the chromomagnetic interaction involve different gyromagnetic factors and short-range correlations. These symmetry-breaking effects mix states with different $\mathrm{SU}(6)_{\text {cs }}$ transformation properties for the $q q$ and $\bar{q} \bar{q}$ pairs, but many of the qualitative features of the symmetry limit remain, both for the hierarchy of masses and decay patterns. However, for detailed phenomenological applications, it is desirable to have explicit estimates of the eigenstates of $H_{\mathrm{CM}}$, and for this purpose, instead of using a basis of $\mathrm{SU}(2)_{\mathrm{s}}, \mathrm{SU}(3)_{\mathrm{c}}$ and $\mathrm{SU}(6)_{\mathrm{cs}}$ representations, it is preferable to couple explicitly the quarks in states of given spin and colour, and similarly for the antiquarks. This new basis turns out also more convenient to impose the constraints due to Pauli principle. The calculations are now carried out in some detail for the scalar, axial and tensor configurations. 


\section{Scalar tetraquarks}

Consider first the case of total spin $S=0$. In the $\left[\left(q_{1} q_{2}\right)\left(\bar{q}_{3} \bar{q}_{4}\right)\right]$ basis, the diquark and the antidiquark should bear conjugate colour, $(\overline{3}, 3)$ or $(6, \overline{6})$, and the same spin 0 or 1 . The Hamiltonian (11) acts on the four states:

$$
\begin{array}{ll}
\phi_{1}=\left(q_{1} q_{2}\right)_{1}^{6} \otimes\left(\bar{q}_{3} \bar{q}_{4}\right)_{1}^{\overline{6}}, \quad \phi_{2}=\left(q_{1} q_{2}\right)_{0}^{\overline{3}} \otimes\left(\bar{q}_{3} \bar{q}_{4}\right)_{0}^{3}, \\
\phi_{3}=\left(q_{1} q_{2}\right)_{0}^{6} \otimes\left(\bar{q}_{3} \bar{q}_{4}\right)_{0}^{\overline{6}}, & \phi_{4}=\left(q_{1} q_{2}\right)_{1}^{\overline{3}} \otimes\left(\bar{q}_{3} \bar{q}_{4}\right)_{1}^{3} .
\end{array}
$$

The colour-magnetic interaction in this basis reads

$$
H_{\mathrm{CM}}=-\left[\begin{array}{ll}
A_{1} & A_{2} \\
B_{1} & B_{2}
\end{array}\right]
$$

with $2 \times 2$ submatrices

$$
\begin{aligned}
& A_{1}=\left[\begin{array}{cc}
\frac{4}{3}\left(C_{34}+C_{12}\right)+\frac{20}{3}\left(C_{14}+C_{13}+C_{23}+C_{24}\right) & 2 \sqrt{6}\left(C_{14}+C_{13}+C_{23}+C_{24}\right) \\
2 \sqrt{6}\left(C_{14}+C_{13}+C_{23}+C_{24}\right) & 8\left(C_{34}+C_{12}\right)
\end{array}\right], \\
& A_{2}=B_{1}^{\dagger}=\frac{2}{\sqrt{3}}\left(C_{13}-C_{14}+C_{24}-C_{23}\right)\left[\begin{array}{cc}
5 & 2 \sqrt{6} \\
0 & 2
\end{array}\right] \text {, } \\
& B_{2}=\left[\begin{array}{cc}
-4\left(C_{34}+C_{12}\right) & 2 \sqrt{6}\left(C_{14}+C_{13}+C_{23}+C_{24}\right) \\
2 \sqrt{6}\left(C_{14}+C_{13}+C_{23}+C_{24}\right) & -\frac{8}{3}\left(C_{34}+C_{12}-C_{14}-C_{13}-C_{23}-C_{24}\right)
\end{array}\right] .
\end{aligned}
$$

In the states $\phi_{1}$ and $\phi_{2}$, the quarks are symmetric in colour-spin and belong to the $(6 \times 6)_{\mathrm{S}}=21$ dimensional representation of $\mathrm{SU}(6)_{\mathrm{cs}}$, and the antiquarks belong to a $\overline{21}$ representation. In $\phi_{3}$ and $\phi_{4}$, the quarks are coupled antisymmetrically in a $(6 \times 6)_{\mathrm{A}}=15$ representation, and the antiquarks in a $\overline{15}$. If only three flavours are involved, $\phi_{1}$ and $\phi_{2}$ fall into the $\overline{3} \times 3=1+8$ representations of $\mathrm{SU}(3)_{\mathrm{F}}$, which is called a nonet in the familiar notation of this symmetry group, and $\phi_{3}$ and $\phi_{4}$ fall into the $6 \times \overline{6}=1+8+27$ representations.

If the two quarks $q_{1}$ and $q_{2}$ or the two antiquarks $\bar{q}_{3}$ and $\bar{q}_{4}$, are identical, the states $\phi_{1}$ and $\phi_{2}$ are excluded by the Pauli principle, and in the space spanned by $\phi_{3}$ and $\phi_{4}$, the Hamiltonian $H_{\mathrm{CM}}$ is expressed by the $2 \times 2$ matrix $-B_{2}$.

In the limit where one antiquark, say $\bar{q}_{4}$, is very heavy and decouples, i.e., $C_{i 4}=C_{4 i}=0$, the problem reduces to the previously discussed [63, 64] chromomagnetic problem of a spin 1/2, colour triplet $(q q \bar{q})$ triquark. It always contains a colour singlet $q \bar{q}$ pair, leading to superallowed decays, if kinematically permitted.

In the flavour-symmetry limit, with the further assumption that the quark-quark and quark-antiquark colourspin interaction strengths are equal, $H_{\mathrm{CM}}$ reduces to

$$
H_{\mathrm{CM}}=-C\left[\begin{array}{cccc}
88 / 3 & 8 \sqrt{6} & 0 & 0 \\
8 \sqrt{6} & 16 & 0 & 0 \\
0 & 0 & -8 & 8 \sqrt{6} \\
0 & 0 & 8 \sqrt{6} & 16 / 3
\end{array}\right]
$$

with eigenvalues $-43.3656 C$ and $-1.9678 C$ in the nonet subspace spanned by $\phi_{1}$ and $\phi_{2}$, and $-19.3656 C$ and $+22.0322 C$ in the 36-plet spanned by $\phi_{3}$ and $\phi_{4}$, which separates out exactly. The lightest state in the nonet and the lightest one in the 36-plet are split by $24 C$, i.e., about $400 \mathrm{MeV}$, exceeding twice the mass difference between strange and non-strange quarks. This led one to predict that the flavour nonet and 36-plet are well separated. It will be shown later that this is not any longer the case, if flavour symmetry is broken also in $H_{\mathrm{CM}}$ (and not only in the constituent masses).

From Eqs. (11-12), it is seen that for the separation of the 36-plet from the nonet to remain, with a blockdiagonal form for $H_{\mathrm{CM}}$, it suffices that $C_{13}=C_{14}$ and $C_{23}=C_{24}$, or $C_{13}=C_{23}$ and $C_{14}=C_{24}$, i.e., both quarks have the same coupling to each antiquarks, or vice-versa. It also persists that the lowest eigenvalue is found in the nonet subspace spanned by $\phi_{1}$ and $\phi_{2}$. 


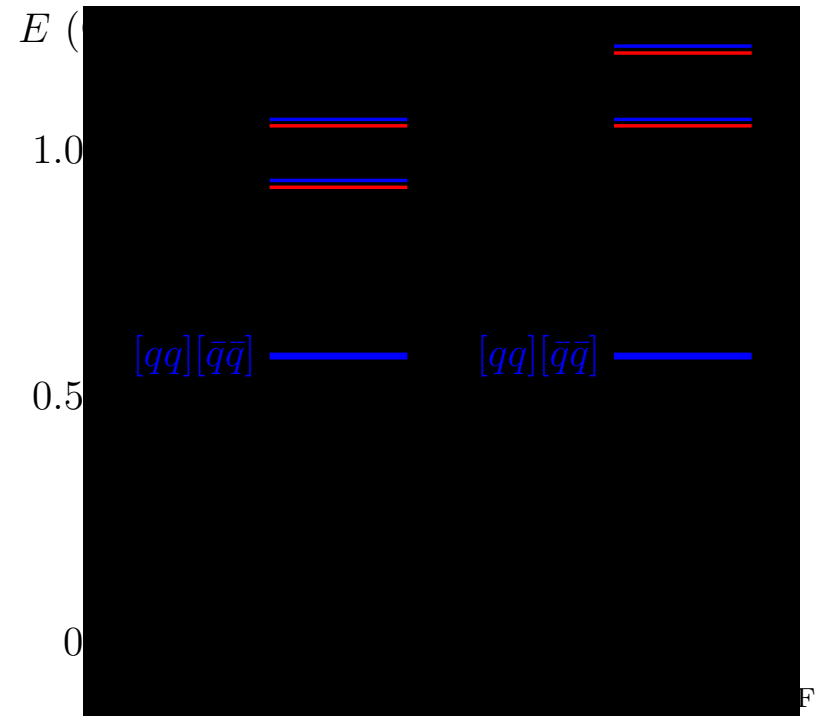

FIG. 1: Spectrum of light multiquark scalars, with a shift $m_{s}-m_{q}$ added for each strange quark or antiquark, and the chromomagnetic term calculated either in the $\mathrm{SU}(\mathrm{N})_{\mathrm{F}}$ limit (left) or with $\mathrm{SU}(\mathrm{N})_{\mathrm{F}}$ breaking (right). In the labels, [qq] denotes the symmetric spin-colour coupling, and $\{q q\}$ the antisymmetric one, and $q=u, d$.

As an illustration, the mass spectrum of light $0^{+}$mesons is shown in Fig. 1 1 with and without flavour symmetry breaking in $H_{\mathrm{CM}}$, with realistic values for the strength factors $C_{i j}$.

For completeness, the crossing matrix is provided between the basis (10) where quarks and antiquarks are paired, and the basis

$$
\begin{array}{ll}
\alpha_{1}=\left(q_{1} \bar{q}_{3}\right)_{0}^{1} \otimes\left(q_{2} \bar{q}_{4}\right)_{0}^{1}, & \alpha_{2}=\left(q_{1} \bar{q}_{3}\right)_{1}^{1} \otimes\left(q_{2} \bar{q}_{4}\right)_{1}^{1}, \\
\alpha_{3}=\left(q_{1} \bar{q}_{3}\right)_{0}^{8} \otimes\left(q_{2} \bar{q}_{4}\right)_{0}^{8}, & \alpha_{4}=\left(q_{1} \bar{q}_{3}\right)_{1}^{8} \otimes\left(q_{2} \bar{q}_{4}\right)_{1}^{8},
\end{array}
$$

with quark-antiquark coupling, it is

$$
\frac{1}{6}\left[\begin{array}{cccc}
3 \sqrt{2} & \sqrt{3} & \sqrt{6} & 3 \\
-\sqrt{6} & 3 & 3 \sqrt{2} & -\sqrt{3} \\
3 & -\sqrt{6} & \sqrt{3} & -3 \sqrt{2} \\
-\sqrt{3} & -3 \sqrt{2} & 3 & \sqrt{6}
\end{array}\right] .
$$

\section{Axial tetraquarks}

The case where the total spin is $S=1$ is somewhat more complicated than the spin $S=0$ case as the recoupling to spin 1 can be done in several ways. The colour-magnetic Hamiltonian now acts over a six-dimensional space with basis

$$
\begin{array}{ll}
\psi_{1}=\left(q_{1} q_{2}\right)_{1}^{6} \otimes\left(\bar{q}_{3} \bar{q}_{4}\right)_{1}^{\overline{6}}, & \psi_{2}=\left(q_{1} q_{2}\right)_{1}^{\overline{3}} \otimes\left(\bar{q}_{3} \bar{q}_{4}\right)_{1}^{3}, \\
\psi_{3}=\left(q_{1} q_{2}\right)_{0}^{\overline{3}} \otimes\left(\bar{q}_{3} \bar{q}_{4}\right)_{1}^{3}, & \psi_{4}=\left(q_{1} q_{2}\right)_{1}^{6} \otimes\left(\bar{q}_{3} \bar{q}_{4}\right)_{0}^{\overline{6}}, \\
\psi_{5}=\left(q_{1} q_{2}\right)_{1}^{\overline{3}} \otimes\left(\bar{q}_{3} \bar{q}_{4}\right)_{0}^{3}, & \psi_{6}=\left(q_{1} q_{2}\right)_{0}^{6} \otimes\left(\bar{q}_{3} \bar{q}_{4}\right)_{1}^{\overline{6}} .
\end{array}
$$

If the two quarks (antiquarks) are identical in flavour, $\psi_{1}, \psi_{3}$ and $\psi_{4}\left(\psi_{1}, \psi_{5}\right.$ and $\left.\psi_{6}\right)$ are excluded by the Pauli principle. This is the case in particular for the manifestly exotic states. 
The colour-magnetic Hamiltonian can be written in terms of $2 \times 2$ blocks as

$$
H_{\mathrm{CM}}=-\left[\begin{array}{lll}
A_{1} & A_{2} & A_{3} \\
B_{1} & B_{2} & B_{3} \\
C_{1} & C_{2} & C_{3}
\end{array}\right]
$$

with

$$
\begin{aligned}
& A_{1}= \frac{2}{3}\left[\begin{array}{cc}
2\left(C_{34}+C_{12}\right)+5\left(C_{14}+C_{13}+C_{23}+C_{24}\right) & 3 \sqrt{2}\left(C_{13}-C_{14}+C_{24}-C_{23}\right) \\
3 \sqrt{2}\left(C_{13}-C_{14}+C_{24}-C_{23}\right) & -4\left(C_{34}+C_{12}\right)+2\left(C_{14}+C_{13}+C_{23}+C_{24}\right)
\end{array}\right], \\
& A_{2}=B_{1}^{\dagger}= \frac{2}{3}\left[\begin{array}{cc}
6\left(C_{13}-C_{14}+C_{23}-C_{24}\right) & -5 \sqrt{2}\left(C_{13}-C_{14}+C_{23}-C_{24}\right) \\
2 \sqrt{2}\left(C_{13}+C_{14}-C_{24}-C_{23}\right) & -6\left(C_{13}+C_{14}-C_{24}-C_{23}\right)
\end{array}\right] \\
& A_{3}=C_{1}^{\dagger}= \frac{2}{3}\left[\begin{array}{cc}
-6\left(C_{13}+C_{14}-C_{24}-C_{23}\right) & 5 \sqrt{2}\left(C_{13}+C_{14}-C_{24}-C_{23}\right) \\
-2 \sqrt{2}\left(C_{13}-C_{14}+C_{23}-C_{24}\right) & 6\left(C_{13}-C_{14}+C_{23}-C_{24}\right)
\end{array}\right] \\
& B_{2}= \frac{2}{3}\left[\begin{array}{cc}
4\left(3 C_{12}-C_{34}\right) & -3 \sqrt{2}\left(C_{13}+C_{14}+C_{23}+C_{24}\right) \\
-3 \sqrt{2}\left(C_{13}+C_{14}+C_{23}+C_{24}\right) & 2 C_{12}-6 C_{34}
\end{array}\right] \\
& B_{3}=C_{2}^{\dagger}=\frac{2}{3}\left[\begin{array}{cc}
-2\left(C_{13}-C_{14}+C_{24}-C_{23}\right) & 0 \\
0 & -5\left(C_{13}-C_{14}+C_{24}-C_{23}\right)
\end{array}\right] \\
& C_{3}=\frac{2}{3}\left[\begin{array}{cc}
-4 C_{12}+12 C_{34} & -3 \sqrt{2}\left(C_{14}+C_{13}+C_{23}+C_{24}\right) \\
-3 \sqrt{2}\left(C_{14}+C_{13}+C_{23}+C_{24}\right) & -6 C_{12}+2 C_{34}
\end{array}\right]
\end{aligned}
$$

In the limit of flavour symmetry where $C_{i j}=C, \forall i, j$, the eigenstates of $H_{\mathrm{CM}}$ have well defined transformation properties under the relevant flavour-symmetry group, and the colour-magnetic Hamiltonian $H_{\mathrm{CM}}$ reduces to the well-known matrix

$$
-\frac{8 C}{3}\left[\begin{array}{cccccc}
6 & 0 & 0 & 0 & 0 & 0 \\
0 & 0 & 0 & 0 & 0 & 0 \\
0 & 0 & 2 & -3 \sqrt{2} & 0 & 0 \\
0 & 0 & -3 \sqrt{2} & -1 & 0 & 0 \\
0 & 0 & 0 & 0 & 2 & -3 \sqrt{2} \\
0 & 0 & 0 & 0 & -3 \sqrt{2} & -1
\end{array}\right]
$$

with eigenvalues $-16 C, 0,-40 C / 3,32 C / 3,-40 C / 3,32 C / 3$. The corresponding flavour multiplets are 9 and 36 for the first eigenvalues, $\overline{18}=\overline{10}+8$ for the next two ones, and $18=10+8$ for the last two ones

Moreover, for the interesting case $(Q Q \bar{u} \bar{d})$ case where the two heavy quarks $Q$ are identical and the two light antiquarks obey isospin symmetry, $H_{\mathrm{CM}}$ also takes the block-diagonal form

$$
-\frac{4}{3}\left[\begin{array}{cccccc}
C_{34}+C_{12}+10 C_{14} & 0 & 0 & 0 & 0 & 0 \\
0 & -2 C_{34}-2 C_{12}+4 C_{14} & 0 & 0 & 0 & 0 \\
0 & 0 & -2 C_{34}+6 C_{12} & -6 \sqrt{2} C_{14} & 0 & 0 \\
0 & 0 & -6 \sqrt{2} C_{14} & C_{12}-3 C_{34} & 0 & 0 \\
0 & 0 & 0 & 0 & -2 C_{12}+6 C_{34} & -6 \sqrt{2} C_{14} \\
0 & 0 & 0 & 0 & -6 \sqrt{2} C_{14} & -3 C_{12}+C_{34}
\end{array}\right]
$$

Note that, contrary to what happens for the spin $S=0$ case, the lowest eigenvalue of the colourmagnetic Hamiltonian survives the Pauli principle, i.e., remains when the basis states $\psi_{1}, \psi_{3}$ and $\psi_{4}$ are removed, at least for all the physically acceptable values of the parameters (see next section).

The crossing matrix from the basis (16) to the basis

$$
\begin{array}{ll}
\beta_{1}=\left(q_{1} \bar{q}_{3}\right)_{0}^{1} \otimes\left(q_{2} \bar{q}_{4}\right)_{1}^{1}, & \beta_{2}=\left(q_{1} \bar{q}_{3}\right)_{1}^{1} \otimes\left(q_{2} \bar{q}_{4}\right)_{0}^{1}, \\
\beta_{3}=\left(q_{1} \bar{q}_{3}\right)_{1}^{1} \otimes\left(q_{2} \bar{q}_{4}\right)_{1}^{1}, & \beta_{4}=\left(q_{1} \bar{q}_{3}\right)_{0}^{8} \otimes\left(q_{2} \bar{q}_{4}\right)_{1}^{8}, \\
\beta_{5}=\left(q_{1} \bar{q}_{3}\right)_{1}^{8} \otimes\left(q_{2} \bar{q}_{4}\right)_{0}^{8}, & \beta_{6}=\left(q_{1} \bar{q}_{3}\right)_{1}^{8} \otimes\left(q_{2} \bar{q}_{4}\right)_{1}^{8},
\end{array}
$$


is

$$
\frac{1}{2 \sqrt{3}}\left[\begin{array}{cccccc}
2 & \sqrt{2} & 1 & -\sqrt{2} & -1 & \sqrt{2} \\
2 & \sqrt{2} & -1 & \sqrt{2} & 1 & -\sqrt{2} \\
0 & 0 & \sqrt{2} & 2 & \sqrt{2} & 2 \\
\sqrt{2} & -2 & -\sqrt{2} & -1 & \sqrt{2} & 1 \\
\sqrt{2} & -2 & \sqrt{2} & 1 & -\sqrt{2} & -1 \\
0 & 0 & -2 & \sqrt{2} & -2 & \sqrt{2}
\end{array}\right] .
$$

\section{E. Tensor tetraquarks}

The survey is ended by the case of spin $S=2$. In the diquark-antidiquark coupling scheme, the chromomagnetic Hamiltonian $H_{\mathrm{CM}}$, written in the basis

$$
\xi_{1}=\left(q_{1} q_{2}\right)_{1}^{6} \otimes\left(\bar{q}_{3} \bar{q}_{4}\right)_{1}^{\overline{6}}, \quad \xi_{2}=\left(q_{1} q_{2}\right)_{1}^{\overline{3}} \otimes\left(\bar{q}_{3} \bar{q}_{4}\right)_{1}^{\overline{3}},
$$

reads

$$
-\frac{2}{3}\left[\begin{array}{cc}
2\left(C_{12}+C_{34}\right)-5\left(C_{13}+C_{24}+C_{14}+C_{23}\right) & -3 \sqrt{2}\left(C_{13}+C_{24}-C_{23}-C_{14}\right) \\
-3 \sqrt{2}\left(C_{13}+C_{24}-C_{23}-C_{14}\right) & -4\left(C_{12}+C_{34}\right)-2\left(C_{13}+C_{24}+C_{23}+C_{14}\right)
\end{array}\right] .
$$

With two quarks identical in flavour, the state $\xi_{1}$ is excluded by the Pauli principle.

As all spins are aligned, the crossing matrix from the basis (24) to the basis

$$
\gamma_{1}=\left(q_{1} \bar{q}_{3}\right)_{1}^{1} \otimes\left(q_{2} \bar{q}_{4}\right)_{1}^{\overline{1}}, \quad \gamma_{2}=\left(q_{1} \bar{q}_{3}\right)_{1}^{8} \otimes\left(q_{2} \bar{q}_{4}\right)_{1}^{\overline{8}},
$$

reduces to the standard crossing matrix of colour

$$
\frac{1}{\sqrt{3}}\left[\begin{array}{cc}
\sqrt{2} & 1 \\
1 & -\sqrt{2}
\end{array}\right]
$$

\section{APPLICATION TO TETRAQUARKS}

This section is devoted to consequences of the chromomagnetic interaction applied to four-quark states for the various flavour configurations.

\section{A. Adjusting the parameters}

The strength parameters $C_{i j}$ for quark-antiquark pairs can be extracted from ordinary mesons, and are given in Table [. They can be considered as upper bounds, as the two-body correlations are stronger in mesons than in tetraquarks. The quark-quark analogues, deduced from the baryon spectrum, are listed in Table II. These parameters can be used to extrapolate the model from ordinary hadrons to multiquarks.

A tempting alternative strategy consists of extracting the parameters from states which are assumed to be dominantly tetraquarks [65] and to apply the model to predict new tetraquark states. However, the observed states very likely result from an intricate mixing of four-quark, two-quark, hybrid and gluonium states, and the fit can be biased if this mixing is ignored.

There is no way to determine the effective masses unambiguously, as they incorporate binding effects which depend on the environment. In particular, the values of $m_{i}$ extracted from baryons are usually higher than those from mesons. This is, indeed, a general property that baryons are heavier, per quark, than mesons, for instance $\mathcal{M}(\Omega) / 3>\mathcal{M}(\phi) / 2$. The inequality $(q q q) / 3>(q \bar{q}) / 2$ can be derived in a large class of models inspired by QCD [66]; in this review article, and refs. therein, it is also reminded that $(q \bar{q})+(Q \bar{Q}) \leq 2(Q \bar{q})$, hence masses deduced from hidden flavour are found lighter than from open flavour. In a multiquark such as $(c \bar{c} q \bar{q})$, a compromise has be found, as in Ref. [15]. 
The chromomagnetic model will thus be used mainly to predict the ordering of the various spin and flavour configurations. Estimating the absolute masses would require a more careful treatment of the chromoelectric effects.

\section{B. Light mesons}

This is the most delicate sector. Experimentally, states are often broad and overlapping. Theoretically, the quark-antiquark spectrum is not as easily described as in the case of heavy quarks, and states with exotic internal structure are thus harder to single out. Moreover, mixing of configurations is more appreciable in this sector. Just to illustrate the complexity, the diagrams with internal $q \bar{q} \leftrightarrow s \bar{s}$ transition through an intermediate gluon (see Fig. 2) mix ten tetraquark states with $I=0$, and six with $I=1$ [57]. Hence great care is required when discussing this sector.

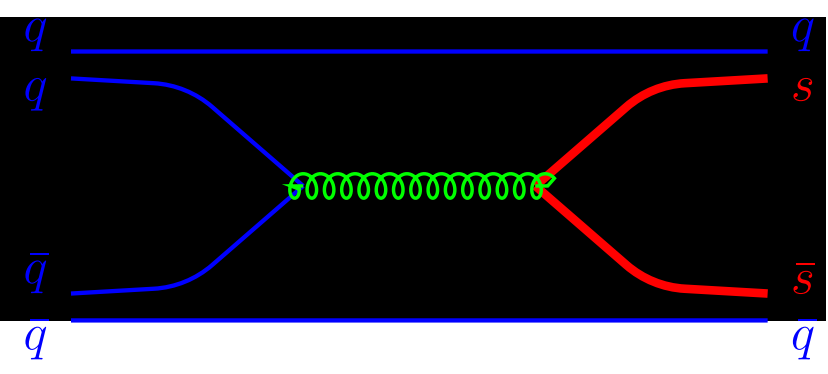

FIG. 2: Example of mixing through internal annihilation

There are many scalar mesons below about $2 \mathrm{GeV}$, and different scenarios have been proposed for their assignment, see, e.g., 51, 52 and references therein. For instance, Klempt et al. 67], using a relativistic quark model and an instanton-induced interaction, proposed that the mainly quark-antiquark multiplet includes $\mathrm{f}^{0}(980)$ and $\mathrm{f}^{0}(1500)$. Alternatively, it is tempting to assign $\mathrm{f}^{0}(1370)$ as being mainly $q \bar{q}$, in its ${ }^{3} \mathrm{P}_{0}$ configuration, which is expected to lie slightly below its ${ }^{3} \mathrm{P}_{1}$ (perhaps mixed with ${ }^{1} \mathrm{P}_{1}$ ) and ${ }^{3} \mathrm{P}_{2}$ partners, in analogy with what is observed in the case of charmonium. This is the point of view adopted, e.g., in [48, 49], where the $\mathrm{f}^{0}(1500)$ is tentatively identified with the lowest gluonium state, the second $q \bar{q}$ state with isospin $I=0$ being slightly higher. The expert view point of the latest issue of the review of particles properties [52] suggests to organize the scalars in a low-lying nonet consisting of $\mathrm{f}^{0}(600), \mathrm{f}^{0}(980), \mathrm{a}(980)$ and $\mathrm{K}_{0}^{*}(800)=\kappa$, a second multiplet being made of $\mathrm{f}^{0}(1370), \mathrm{f}^{0}(1500), \mathrm{a}(1450)$ and $\mathrm{K}_{0}^{*}(1430)$, with the caveat that the $\mathrm{f}^{0}(1500)$ is copiously mixed with the $\mathrm{f}^{0}(1710)$ to share their $q \bar{q}, s \bar{s}$ and gluonium content. This is not too far from the recent analysis by Narison 68 .

If it is assumed that $C_{q q}=C_{q \bar{q}}, C_{q s}=C_{q \bar{s}}=0.625 C_{q q}$, and $C_{s s}=C_{s \bar{s}}=C_{q s}^{2} / C_{q q}$, and if the values $C_{q q}=19.2 \mathrm{MeV}, m_{q}=320 \mathrm{MeV}$ and $m_{s}=445 \mathrm{MeV}$ are adopted, the two above nonets come with masses (439, $722,980) \mathrm{MeV}$ and $(1242,1376,1512) \mathrm{MeV}$, respectively, in our simple chromomagnetic model. The agreement with experimental masses is perhaps too good, as mixing with other configurations is expected to shift these results. Anyhow, these parameters also give the remaining tetraquark spectrum. In particular, for the $Y=2$ axials considered in [65] with $(q q \bar{s} \bar{s})$ content, a first state is found at $1310 \mathrm{MeV}$ and a heavier one at $1620 \mathrm{MeV}$, while for the $27(I=1) Y=2$, the mass is predicted to be about $1540 \mathrm{MeV}$. States with both open and hidden strangeness are obtained at 1510 and $1870 \mathrm{Mev}\left(0^{+}\right), 1500,1640$ and $1760 \mathrm{MeV}\left(1^{+}\right)$and $1810 \mathrm{MeV}\left(2^{+}\right)$, and an axial state with a double hidden strangeness is predicted at $1780 \mathrm{MeV}$. Moreover, the $\phi \omega$ resonance found at BES II [69] at $1812 \mathrm{MeV}$ could be identified with the multiquark scalar with hidden strangeness.

A difficulty with this description of tetraquarks, or at least, with this choice of parameters, is the prediction of a scalar multiplet with $I=0,1,2$ at about $800 \mathrm{MeV}$, without experimental evidence for a $I=1$ resonance nor for a $I=2$ one in that region. ${ }^{1}$ Also, a puzzlingly light $I=0$ state would be predicted with this set of parameters.

1 Note that the repulsive character of the $I=2 \pi \pi$ phase-shift in this region is not a definite obstacle, as well the pentaquark is 
An alternative strategy consists of adopting the parameters $m_{i}$ and $C_{i j}$ deduced from baryon masses. With this choice of parameters, the main message is conveyed by Fig. 1. There is an isolated isoscalar which can be identified with $\sigma$. Next comes a nearly degenerate set of $I=0, I=1$ and $I=2$ states corresponding to $f^{0}(980)$, a $(980)$ and a yet not discovered $I=2$ state. The states with one unit of strangeness correspond to $\kappa$. The first state with hidden strangeness is at about $1050 \mathrm{MeV}$.

Note that with $C_{q s}<C_{q q}$, which is expected as a consequence of flavour symmetry breaking, the states with hidden strangeness will be pushed up in mass relative to their mass in the flavour symmetric case. Indeed as can be seen from Fig. 1, with that choice of parameters, the states with hidden strangeness $(I=0$ and $I=1)$ in the flavour nonet is more massive than the $I=0,1$ and 2 states without hidden strangeness in the flavour 36-plet! A bag model calculation found these nonet and 36-plet states at almost the same mass and the authors considered their mixing with interesting results $[70]^{2}$. This is at significant variance with the results of [26], which inspired several phenomenological analyses. In short, the $\mathrm{a}_{0}$ and $\mathrm{f}_{0}$, if described as multiquark hadrons, do no acquire much hidden strangeness from short-range, direct quark interaction. Indeed, the chromomagnetic effects are significantly weaker for strange quarks than for ordinary quarks. The mixing is particularly important for light scalar mesons with $I=0$ and, to a lesser extent $I=1$, because the wave function obtained by diagonalisation of $H_{\mathrm{CM}}$ contains large components where a quark-antiquark pair has spin triplet and colour octet.

The above discussion suggests that it is diffcult to explain the low-lying $0^{+}$mesons as tetraquark states without mixing to other configurations, even if flavour-symmetry breaking is treated consistently in the tetraquark sector. One should note the inverted nonet of light scalar mesons, that comes out naturally from the chromomagnetic interaction in light tetraquarks, also arises in a multichannel picture where colour-singlet quark-antiquark states are coupled to meson-meson channels [72, 73, 74]. This is also the conclusion of Maiani et al. [75] that light tetraquark scalars lie below the quark-antiquark ones.

\section{Mesons with heavy flavour}

This sector has been particularly discussed recently, following the observation by several groups of the $\mathrm{D}_{s}(2317)$ and $\mathrm{D}_{s}(2457)$ resonances with $(c \bar{s})$ flavour content 52]. The current wisdom is that these states have $J^{P}=0^{+}$and $1^{+}$quantum numbers, respectively. In standard quark models, see, e.g., 76], the masses of the ${ }^{3} \mathrm{P}_{0}$ and ${ }^{3} \mathrm{P}_{1}$ (perhaps mixed with ${ }^{1} \mathrm{P}_{1}$ ) ground states are, by about $100 \mathrm{MeV}$ too high to match the observed masses, and the $\mathrm{D}_{s, J}$ states are therefore to be described with other tools. See, however, 77, 78], where the new states with open charm are accommodated without a need for any exotic structure.

The fashionable "molecular picture" has been proposed for these states, and was even anticipated before their discovery, see, e.g., 79, 80] and references there. In another scenario, these states are described as chiral partners of the pseudoscalar $\mathrm{D}_{s}(1968)$ and vector $\mathrm{D}_{s}(2112)$ ground states. Again, the restoration of chiral symmetry in this sector was predicted as reminded in the recent analysis 81] where references are given to the earlier papers. The doubling of $\mathrm{D}_{s}$ states by chiral symmetry, is, however, not universally accepted, as discussed, e.g., 82].

If the $\mathrm{D}_{s}(2317)$ and $\mathrm{D}_{s}(2457)$ are basically four-quark states, as suggested, e.g., in [83, 84], then the states with dominant quark-antiquark content bearing the same quantum numbers await experimental discovery.

In the calculation based on our chromomagnetic Hamiltonian and our preferred choice of parameters, the $0^{+}$ and $1^{+}$multiquark states come too high as compared to the experimental masses and are thus rather broad. Still, they produce a mixing with the $0^{+}$and $1^{+}$states of $(c \bar{s})$ whose masses are thus pushed down. In short, the $\mathrm{D}_{s}(2317)$ and $\mathrm{D}_{s}(2457)$ states are perhaps quark-antiquark states mixed with four-quark states and hence lighter than predicted in simple quark models. A similar scenario was suggested, e.g., in [84, 85, 86].

not ruled out solely by the negative values of the $\mathrm{KN}$ phase-shift. If one imagines a hadron-hadron potential with a repulsive long-range tail and an attractive short-range well due to quark dynamics, low-energy scattering experiments will hardly detect the presence of the inner hole and will only feel the repulsive tail.

2 To us it seems that this mixing could be more important than the mixing between $0^{+}$states inside the flavour nonet estimated by F. Giacosa et. al. [71]. 


\section{Mesons with heavy hidden flavour}

There is already a long history of possible multiquarks with hidden charm, and any new candidate is examined with precaution. In the late 70's, the molecular charmonium was proposed [5, 87] for high-lying $\psi(4.028)$ or $\psi(4.414)$ states, to account for their narrowness or for the deviation from spin counting rules for the relative branching ratios to $\mathrm{D} \overline{\mathrm{D}}, \mathrm{D} \overline{\mathrm{D}}^{*}+$ c.c. and $\mathrm{D}^{*} \overline{\mathrm{D}}^{*}$. However, these decay properties were explained by the node structure of these states being mostly $(c \bar{c}) n^{3} \mathrm{~S}_{1}$ with radial number $n=3$ or $n=4$ [88, 89, 90].

More recently, the states X(3872), X(3940) and Y(4260) were found in B-factories, and at least the X(3872) was confirmed elsewhere [3, 4]. Scrutinising the mass, decay properties and production mechanism does not lead to a fully convincing $n^{2 s+1} L_{J}$ assignment in the spectrum of charmonium. Hence other structures have been proposed. A $\bar{D}^{*}+$ c.c. molecular structure is particularly tempting for the $\mathrm{X}(3872)$, since it lies just above this threshold, and binding or near binding of this system was predicted on the basis of long-range nuclear interaction between $\mathrm{D}$ and $\overline{\mathrm{D}}^{*}$. For references, see [8, 9, 10, 91, 92], where the $\mathrm{X}(3872)$ is analysed, and also the comments by Susuki on this picture 11].

Another possibility is that one of the $\mathrm{X}(3872), \mathrm{X}(3940)$ and $\mathrm{Y}(4260)$ resonances is a state of the long-awaited charmonium hybrid, schematically denoted $(c \bar{c} g)$. Already in the 70's and early 80 's, one speculated about some new kind of states in mesons and baryons, where the string linking quarks, or the gluon field in the QCD language, is excited, see, e.g., Ref. [93, 94, 95], followed by several studies in the framework of models with constituent gluons or flux tube, or within QCD sum rules or lattice QCD. The possibility that one of the new hidden-charm meson is an hybrid is discussed, e.g., in [96, 97]. The isospin violation observed in the $\mathrm{X}(3872)$ is hardly explained in the hybrid scenario. On the other hand, the hybrid interpretation of the Y(4260) is better supported by the data, as stressed in the recent analysis by the CLEO collaboration [98].

As neither the molecular nor the hybrid interpretation has won an overall consensus, yet, the door remains open for a four-quark interpretation $(c \bar{c} q \bar{q})$ or $(c \bar{c} s \bar{s})$. Maiani et al., in particular, suggested a diquarkantidiquark picture of these states, for instance $(c s)(\bar{c} \bar{s})$ for the $\mathrm{Y}(4260)$ [99]. The effective mass of the diquark, in this approach, accounts for the strong quark-quark correlation, and the (cs) subsystem is restricted to have colour $\overline{3}$ and spin $s=0$. In the calculation presented here, any subsystem has all the possible quantum numbers compatible with a given $J^{P C}$ hypothesis for the whole system, and the weight of each configuration is determined by diagonalising the chromomagnetic interaction.

As already discussed in [15], this procedure highlights a remarkable eigenstate, which is a pure $(c \bar{c})_{8}(q \bar{q})_{8}$ octet-octet of colour in the limit where $C_{c q}=C_{c \bar{q}}$, and has a small $(c \bar{c})_{1}(q \bar{q})_{1}$ singlet-singlet component if this condition is broken, restricted however to $(s=1) \otimes(s=1)$ for the spins, explaining the observed $\mathrm{J} / \psi+\omega$ and $\mathrm{J} / \psi+\pi^{+} \pi^{-}$modes and the absence of $\mathrm{J} / \psi+$ pseudoscalar. In the crossed rearrangement $(c \bar{q})(\bar{c} q)$ of the constituents, the colour content is dominantly singlet-singlet, but this is $\mathrm{D}^{*}+$ c.c., which is suppressed by the lack of phase-space. The chromomagnetic mechanism for tetraquarks with hidden charm has been further studied by Stancu [100].

As for the $X_{b}(b \bar{b} q \bar{q})$, the analogue of $X(3872)$ in the hidden-beauty sector, if the parameters are tuned to fit the measured values of the masses of $\mathrm{B}, \mathrm{B}^{*}, \Upsilon$ and $\Lambda_{b}$ hadrons, it appears to be stable against dissociation into $\mathrm{B}^{*}$, with, however, possible decay into $\Upsilon+\omega[15]$.

\section{E. Mesons with double heavy flavour}

It often happens in the field of exotic hadrons that a new calculation of a given configuration gives results at variance or in serious conflict with the previous ones. An example is pentaquark with flavour content (uudd $\bar{s}$ ) or its heavier analogue with $s$ replaced by $c$, found either unbound or nearly stable, and in the latter case, either with positive or negative parity. An exception is the sector of $\left(Q Q^{\prime} \bar{q} \bar{q}^{\prime}\right)$ states with two heavy quarks and two light antiquarks, or vice-versa. An abundant literature has been accumulated over the years for these exotic configurations [18, 19, 20, 21, 22, 23, 24, 25]. All the papers convey basically the same message, namely if the mass ratio $M / m$ of quarks to antiquarks is large enough, this state becomes stable against spontaneous dissociation into $(Q \bar{q})+\left(Q^{\prime} \bar{q}^{\prime}\right)$, which is the lowest threshold if $Q^{\prime}$ is heavier than $Q$ and $q^{\prime}$ than $q$. If $Q \neq Q^{\prime}$, $M$ can be taken as twice their reduced mass.

This binding occurs for a spin- and flavour-independent interaction, and can be said to be of chromoelectric nature. The same effect is observed in atomic physics (see, e.g., [17] for references) for a system of four unit 
charges $\left(M^{+}, M^{+}, m^{-}, m^{-}\right)$: whilst the systems with equal masses, which corresponds to the positronium molecule $\mathrm{Ps}_{2}$, is just by a small amount below the threshold for dissociation into two positronium atoms, the hydrogen molecule, for which $M \gg m$, is more deeply bound and possesses a rich spectrum of excitations.

In model calculations, mesons with double charm, corresponding to the case of $Q=Q^{\prime}=c$, appear at best as marginally bound [25], and are most often found to be unbound. It is difficult to say whether the groundstate is actually unstable in the assumed model, a better treatment of the 4-body problem might change the conclusions. It is thus important to analyse to which extent the chromomagnetic interaction can help achieving the binding, or has a repulsive effect.

For $(Q Q \bar{q} \bar{q})$ with identical heavy quarks, the chromomagnetic interaction is optimal for $J^{P}=1^{+}$, since the Pauli principle forbids the $0^{+}$eigenstates with the lowest eigenvalue of $H_{\mathrm{CM}}$. This restriction does not apply to states with charm and beauty, and the most favourable situation occurs to be $(b c \bar{u} \bar{d})$ with $J^{P}=0^{+}$and isospin $I=0$. The very large value of the mass ratio $M_{Q} / m_{n}$, where $M_{Q}^{-1}$ is the average of the inverse masses $m_{c}$ and $m_{b}{ }^{3}$, presumably gives binding or almost binding from the sole chromoelectric effects. The chromomagnetic interaction is also favourable, and, if alone, would give a binding of more than $100 \mathrm{MeV}$.

Of course, it would be naive to add this chromomagnetic binding energy to the chromoelectric binding estimated in another model. Each term, to provide with the best attraction, require a specific internal spin-colour function, and with the two interaction terms switched on, a compromise has to be found. It is however reasonable to believe that the net energy would lie below the lowest of the chromoelectric and the chromomagnetic ones.

A search for this exotic meson with charm $C=1$ and beauty $B=-1$ does not seem out of reach. The ground state of $(b \bar{c})$ has already been found [52] and the ground state of $(c c q)$ has been observed in an experiment [101, 102], though not confirmed in others [103]. Moreover, double-charm production has been observed at beauty factories, permitting in particular to identify new charmonia recoiling against the $\mathrm{J} / \psi$ [104]. Hence other systems with two units of heavy flavour should be accessible. Also, the potential of heavy-ion collisions as a flavour factory to produce exotic hadrons [105] has not yet been fully exploited.

\section{OUTLOOK}

A survey of various flavour configurations show that the spectrum of the lowest positive-parity mesons is greatly influenced by multiquark configurations. For the crypto-exotic states, a detailed comparison with experimental data cannot avoid a delicate mixing scheme involving orbitally excited quark-antiquark states (including radial excitation) and hybrids, and presumably glueball for the case of zero isospin and strangeness. The analysis presented here shows that the multiquark component has a level ordering somewhat different of the one currently used, with scalar $(q \bar{q} s \bar{s})$ mesons higher in the spectrum. The possibility of a spin $S=2$, isospin $I=2$ resonance should be considered seriously. There are, indeed, indications [106] that this exotic state might be in the recent experimental data of the L3 collaboration [107], and in earlier $\gamma \gamma$ data in gamma-gamma reactions 108, 109, 110, 111]

In the sector of naked charm $C=1$, the dynamical scheme presented here does not permit to assign the $\mathrm{D}_{s, J}$ states to be the $J^{P}=0^{+}$and $1^{+}$states of $(c \bar{s} n \bar{n})$. A tricky possibility, however, exists, that these multiquarks are broad but lie not too far and hence that the $0^{+}$and $1^{+}$states of $(c \bar{s})$ are pushed down by mixing.

In the sector of charm $C=2$, or in the sector with charm $C=1$ and beauty $B=-1$, a serious multiquark candidate is $(b c \bar{u} \bar{d})$ with $J^{P}=1^{+}$. The main reason is the tendency for heavy quarks to experience the best binding in a given -flavour independent - confining well, i.e., the binding is essentially chromoelectric. However, the chromomagnetic interaction helps.

In the more accessible sector with hidden charm, the $\mathrm{X}(3872)$ is well described as an eigenstate of the chromomagnetic interaction, which is basically a pure octet-octet $\left[(c \bar{c})_{8}(q \bar{q})_{8}\right]$, and a small impurity which explains the data on the $\mathrm{J} / \psi \pi^{+} \pi^{-}$decay. In the other coupling scheme, the $\mathrm{X}(3872)$ is largely of singlet-singlet type, i.e., $\left[(c \bar{q})_{1}(\bar{c} q)_{1}\right.$, but as $\mathrm{D} \overline{\mathrm{D}}$ is forbidden, the decay proceeds via $\mathrm{D}^{*}+$ c.c. and lacks phase-space. This

\footnotetext{
3 The inverse masses, entering linearly the Schrödinger equation are more pertinent that the masses themselves to follow the variation of the binding energy
} 
explains the remarkably small width of this state. This picture raises interesting questions. The quark model predicts degenerate isospin states, $I=0$ and $I=1$. The neutral members mix by annihilation diagrams, and the narrowest shows up most strikingly in the data. The charged members of the $I=1$ multiplet are perhaps not so easily produced as the neutral in B decay, and should be searched for by other production mechanisms. The direct treatment of the four-quark interaction differs from the nuclear-physics approach, which used the well identified D and $\mathrm{D}^{*}$ mesons, and the well known Yukawa mechanism of long-range interaction between hadrons. However, in absence of evidence for a repulsive core keeping the hadrons well separated, the situation is different from that of nucleons within a nucleus, and it is not sure whether or not the bound states dynamics remains dominated by long-range forces.

It is worth stressing once more that a four-quark explanation of a few remarkable mesons such as the $X(3872)$ does not imply an abundance of multiquark states in the forthcoming experimental data. Most S-wave multiquarks are very broad, as they spontaneously split into two colour-singlet hadrons. Only the states with very low mass or peculiar internal structure survive this dissociation and show up as narrow peaks.

\section{Acknowledgments}

H.H. thanks the LAPTH, Annecy-le-Vieux, and the CERN Particle Theory Unit for their kind hospitality. The comments by M. Asghar on the manuscript are gratefully acknowledged.

[1] E. Klempt, AIP Conf. Proc. 814, 723 (2006).

[2] T. Barnes, AIP Conf. Proc. 814, 735 (2006), hep-ph/0510365.

[3] S. Jin, Int. J. Mod. Phys. A20, 5145 (2005).

[4] E. S. Swanson, Phys. Rept. 429, 243 (2006), hep-ph/0601110.

[5] M. B. Voloshin and L. B. Okun, JETP Lett. 23, 333 (1976).

[6] N. A. Tornqvist, Phys. Rev. Lett. 67, 556 (1991).

[7] T. E. O. Ericson and G. Karl, Phys. Lett. B309, 426 (1993).

[8] E. Braaten and M. Kusunoki, Phys. Rev. D72, 054022 (2005), hep-ph/0507163.

[9] E. S. Swanson, Phys. Lett. B588, 189 (2004), hep-ph/0311229.

[10] F. E. Close and P. R. Page, Phys. Lett. B578, 119 (2004), hep-ph/0309253.

[11] M. Suzuki, Phys. Rev. D72, 114013 (2005), hep-ph/0508258.

[12] Y. S. Kalashnikova, Phys. Rev. D72, 034010 (2005), hep-ph/0506270.

[13] S. K. Choi et al. (Belle), Phys. Rev. Lett. 91, 262001 (2003), hep-ex/0309032.

[14] L. Maiani, F. Piccinini, A. D. Polosa, and V. Riquer, Phys. Rev. D71, 014028 (2005), hep-ph/0412098.

[15] H. Hogaasen, J. M. Richard, and P. Sorba, Phys. Rev. D73, 054013 (2006), hep-ph/0511039.

[16] B. Julia-Diaz and D. O. Riska, Nucl. Phys. A755, 431 (2005), nucl-th/0405061.

[17] E. Armour, J.-M. Richard, and K. Varga, Phys. Rept. 413, 1 (2005), physics/0411204.

[18] J. P. Ader, J. M. Richard, and P. Taxil, Phys. Rev. D25, 2370 (1982).

[19] S. Zouzou, B. Silvestre-Brac, C. Gignoux, and J. M. Richard, Z. Phys. C30, 457 (1986).

[20] L. Heller and J. A. Tjon, Phys. Rev. D35, 969 (1987).

[21] J. Carlson, L. Heller, and J. A. Tjon, Phys. Rev. D37, 744 (1988).

[22] H. J. Lipkin, Phys. Lett. B172, 242 (1986).

[23] D. M. Brink and F. Stancu, Phys. Rev. D57, 6778 (1998).

[24] B. A. Gelman and S. Nussinov, Phys. Lett. B551, 296 (2003), hep-ph/0209095.

[25] D. Janc and M. Rosina, Few Body Syst. 35, 175 (2004), hep-ph/0405208.

[26] R. L. Jaffe, Phys. Rev. D15, 267 (1977).

[27] R. L. Jaffe, Phys. Rev. D15, 281 (1977).

[28] R. L. Jaffe, Phys. Rev. Lett. 38, 195 (1977).

[29] K. Yamamoto et al. (E885), Phys. Lett. B478, 401 (2000).

[30] C. Gignoux, B. Silvestre-Brac, and J. M. Richard, Phys. Lett. B193, 323 (1987).

[31] H. J. Lipkin, Phys. Lett. B195, 484 (1987).

[32] B. Silvestre-Brac and J. Leandri, Phys. Rev. D45, 4221 (1992).

[33] J. Leandri and B. Silvestre-Brac, Phys. Rev. D40, 2340 (1989).

[34] D. B. Lichtenberg and R. Roncaglia (1992), presented at Int. Workshop on Diquarks, Turin, Italy, Nov 2-4, 1992. 
[35] L. Montanet, G. C. Rossi, and G. Veneziano, Phys. Rept. 63, 149 (1980).

[36] K. Johnson and C. B. Thorn, Phys. Rev. D13, 1934 (1976).

[37] H.-M. Chan and H. Hogaasen, Phys. Lett. B72, 121 (1977).

[38] H.-M. Chan et al., Phys. Lett. B76, 634 (1978).

[39] H. Hogaasen and P. Sorba, Nucl. Phys. B145, 119 (1978).

[40] J. L. Rosner, Phys. Rev. D33, 2043 (1986).

[41] G. Karl and P. Zenczykowski, Phys. Rev. D36, 2079 (1987).

[42] G. Karl and P. Zenczykowski, Phys. Rev. D36, 3520 (1987).

[43] T. Sakai, K. Shimizu, and K. Yazaki, Prog. Theor. Phys. Suppl. 137, 121 (2000), nucl-th/9912063.

[44] F. Stancu (1999), published in Bled 1999, Hadrons as solitons 65-66, ed. M. Rosina, see http://www-f1.ijs.si/Bled2000/previous/R_proc.html, hep-ph/9910547.

[45] K. Abe et al. (Belle), Phys. Rev. Lett. 94, 182002 (2005), hep-ex/0408126.

[46] B. Aubert et al. (BABAR), Phys. Rev. Lett. 95, 142001 (2005), hep-ex/0506081.

[47] N. Isgur and H. J. Lipkin, Phys. Lett. B99, 151 (1981).

[48] C. Amsler and F. E. Close, Phys. Lett. B353, 385 (1995), hep-ph/9505219.

[49] C. Amsler and F. E. Close, Phys. Rev. D53, 295 (1996), hep-ph/9507326.

[50] F. E. Close and N. A. Tornqvist, J. Phys. G28, R249 (2002), hep-ph/0204205.

[51] C. Amsler and N. A. Tornqvist, Phys. Rept. 389, 61 (2004).

[52] S. Eidelman et al. (Particle Data Group), Phys. Lett. B592, 1 (2004).

[53] A. De Rujula, H. Georgi, and S. L. Glashow, Phys. Rev. D12, 147 (1975).

[54] N. Isgur and H. B. Thacker, Phys. Rev. D64, 094507 (2001).

[55] E. J. Eichten and C. Quigg, Phys. Rev. D49, 5845 (1994), hep-ph/9402210.

[56] S. Fleck and J. M. Richard, Prog. Theor. Phys. 82, 760 (1989).

[57] H.-M. Chan and H. Hogaasen, Zeit. Phys. C7, 25 (1980).

[58] C. W. Wong and K. F. Liu, Phys. Rev. D21, 2039 (1980).

[59] P. J. G. Mulders, A. T. M. Aerts, and J. J. de Swart (1978), submitted to 19th Int. Conf. on High Energy Physics, Tokyo, Japan, Aug 23-30, 1978.

[60] H.R. Petry, in Lecture Notes in Physics, Vol. 197 (Springer-Verlag, 1983) p. 459.

[61] K. Bleuler, in Perspectives in nuclear physics at intermediate energies, Edited by S. Boffi, C. Ciofi degli Atti, M.M. Giannini. (Workshop on Perspectives in Nuclear Physics at Intermediate Energies, Trieste, Italy, Oct 10-14, 1983). 1984.

[62] F. Buccella, Mod. Phys. Lett. A21, 831 (2006), hep-ph/0411244.

[63] H. Hogaasen and P. Sorba, Mod. Phys. Lett. A19, 2403 (2004), hep-ph/0406078.

[64] H. Hogaasen and P. Sorba, Fizika B 14, 245 (2005), hep-ph/0410224.

[65] H. X. Chen, A. Hosaka and S. L. Zhu, Phys. Rev. D 74, 054001 (2006) arXiv:hep-ph/0604049.

[66] S. Nussinov and M. A. Lampert, Phys. Rept. 362, 193 (2002), hep-ph/9911532.

[67] E. Klempt, B. C. Metsch, C. R. Munz, and H. R. Petry, Phys. Lett. B361, 160 (1995), hep-ph/9507449.

[68] S. Narison, Phys. Rev. D 73 (2006) 114024 arXiv:hep-ph/0512256.

[69] M. Ablikim et al. (BES), Phys. Rev. Lett. 96, 162002 (2006), hep-ex/0602031.

[70] R. P. Bickerstaff and B. H. J. McKellar, Z. Phys. C16, 171 (1982).

[71] F. Giacosa, Phys. Rev. D 74, 014028 (2006) arXiv:hep-ph/0605191.

[72] E. Van Beveren et al., Z. Phys. C30, 615 (1986).

[73] E. van Beveren, D. V. Bugg, F. Kleefeld and G. Rupp, Phys. Lett. B 641, 265 (2006) arXiv:hep-ph/0606022.

[74] N. A. Tornqvist, Z. Phys. C68, 647 (1995), hep-ph/9504372.

[75] L. Maiani, F. Piccinini, A. D. Polosa, and V. Riquer (2006), hep-ph/0604018.

[76] R. N. Cahn and J. D. Jackson, Phys. Rev. D68, 037502 (2003), hep-ph/0305012.

[77] A. Deandrea, G. Nardulli, and A. D. Polosa, Phys. Rev. D68, 097501 (2003), hep-ph/0307069.

[78] T. Matsuki, T. Morii, and K. Sudoh (2006), hep-ph/0605019.

[79] T. Barnes, F. E. Close, and H. J. Lipkin, Phys. Rev. D68, 054006 (2003), hep-ph/0305025.

[80] P. Bicudo, Nucl. Phys. A748, 537 (2005), hep-ph/0401106.

[81] W. A. Bardeen, E. J. Eichten, and C. T. Hill, Phys. Rev. D68, 054024 (2003), hep-ph/0305049.

[82] R. L. Jaffe, D. Pirjol, and A. Scardicchio, Phys. Rev. Lett. 96, 121601 (2006), hep-ph/0511081.

[83] H. J. Lipkin, Phys. Lett. B580, 50 (2004), hep-ph/0306204.

[84] K. Terasaki and B. H. J. McKellar, Prog. Theor. Phys. 114, 205 (2005), hep-ph/0501188.

[85] T. E. Browder, S. Pakvasa, and A. A. Petrov, Phys. Lett. B578, 365 (2004), hep-ph/0307054.

[86] E. van Beveren and G. Rupp, Phys. Rev. Lett. 91, 012003 (2003), hep-ph/0305035.

[87] A. De Rujula, H. Georgi, and S. L. Glashow, Phys. Rev. Lett. 38, 317 (1977).

[88] A. Le Yaouanc, L. Oliver, O. Pene, and J. C. Raynal, Phys. Lett. B71, 397 (1977).

[89] A. Le Yaouanc, L. Oliver, O. Pene, and J. C. Raynal, Phys. Lett. B72, 57 (1977). 
[90] E. Eichten, K. Gottfried, T. Kinoshita, K. D. Lane, and T.-M. Yan, Phys. Rev. D21, 203 (1980).

[91] M. B. Voloshin, Phys. Lett. B604, 69 (2004), hep-ph/0408321.

[92] N. A. Tornqvist, Phys. Lett. B590, 209 (2004), hep-ph/0402237.

[93] R. Giles and S. H. H. Tye, Phys. Rev. D16, 1079 (1977).

[94] D. Horn and J. Mandula, Phys. Rev. D17, 898 (1978).

[95] P. Hasenfratz, R. R. Horgan, J. Kuti, and J. M. Richard, Phys. Lett. B95, 299 (1980).

[96] E. Kou and O. Pene, Phys. Lett. B631, 164 (2005), hep-ph/0507119.

[97] F. E. Close and P. R. Page, Phys. Lett. B628, 215 (2005), hep-ph/0507199.

[98] T. E. Coan et al. (CLEO), Phys. Rev. Lett. 96, 162003 (2006), hep-ex/0602034.

[99] L. Maiani, V. Riquer, F. Piccinini, and A. D. Polosa, Phys. Rev. D72, 031502 (2005), hep-ph/0507062.

[100] F. Stancu (2006), hep-ph/0607077.

[101] M. Mattson et al. (SELEX), Phys. Rev. Lett. 89, 112001 (2002), hep-ex/0208014.

[102] A. Ocherashvili et al. (SELEX), Phys. Lett. B628, 18 (2005), hep-ex/0406033.

[103] B. Aubert (BABAR) (2006), hep-ex/0605075.

[104] S. K. Choi et al. (BELLE), Phys. Rev. Lett. 89, 102001, 129901(E) (2002), hep-ex/0206002.

[105] S. Kabana (STAR), Acta Phys. Hung. A24, 321 (2005), hep-ex/0406032.

[106] I. V. Anikin, B. Pire, and O. V. Teryaev, Phys. Lett. B626, 86 (2005), hep-ph/0506277.

[107] P. Achard et al. [L3 Collaboration], Phys. Lett. B 638, 128 (2006) arXiv:hep-ex/0605021].

[108] B. A. Li and K. F. Liu, Phys. Lett. B118, 435 (1982), [Erratum-ibid. B 124 (1983) 550].

[109] K. F. Liu and B. A. Li, Phys. Rev. Lett. 58, 2288 (1987).

[110] N. N. Achasov, S. A. Devyanin, and G. N. Shestakov, Phys. Lett. B108, 134 (1982), [Erratum-ibid. B 108 (1982) 435].

[111] N. N. Achasov and G. N. Shestakov, Phys. Lett. B203, 309 (1988). 